\title{
L'ordre public des religions : un itinéraire
}

\author{
Pierre-Henri Prélot
}

\section{(2penEdition}

\section{Journals}

Édition électronique

URL : http://journals.openedition.org/rdr/1112

DOI : $10.4000 /$ rdr 1112

ISSN : 2534-7462

\section{Éditeur}

Presses universitaires de Strasbourg

\section{Édition imprimée}

Date de publication : 9 juillet 2020

Pagination : $59-72$

ISBN : $979-10-344-0065-2$

ISSN : 2493-8637

\section{Référence électronique}

Pierre-Henri Prélot, «L'ordre public des religions : un itinéraire », Revue du droit des religions [En ligne] 9 | 2020, mis en ligne le 18 mai 2020, consulté le 20 novembre 2020. URL : http:// journals.openedition.org/rdr/1112; DOI : https://doi.org/10.4000/rdr.1112

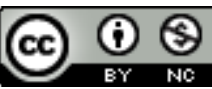

La revue du droit des religions est mise à disposition selon les termes de la Creative Commons Attribution - Pas d'Utilisation Commerciale 4.0 International - CC BY-NC 4.0. 


\section{L'ORDRE PUBLICDESRELIGIONS: UNITINÉRAIRE}

\section{Pierre-Henri PRÉLOT}

Université de Cergy-Pontoise

\section{Résumé}

Entrée dans le droit moderne avec l'article 10 de la Déclaration des droits de l'homme et du citoyen en rapport avec la liberté de religion, la notion d'ordre public va faire un retour en force deux siècles plus tard à l'occasion des débats relatifs à la présence des religions dans l'espace social. Le contenu de l'ordre public va alors connaître une extension considérable, puisqu'au-delà de l'ordre public purement «matériel» qui est l'objet traditionnel du pouvoir de police, il inclut désormais les «exigences minimales de la vie en société» et le «vivre ensemble», ce qui tend à démontrer que la liberté de religion n’a jamais été considérée comme une liberté ordinaire parmi les droits que notre Constitution garantit.

\section{Abstract}

The concept of public order was introduced into modern law with Article 10 of the Declaration of the Rights of Man and of the Citizen in relation to freedom of religion. Two centuries later, the notion of public order has made a strong comeback in the debates on the presence of religions in society. The content of public order has been considerably extended. Beyond the purely "material" public order which is the traditional aim of policing power, it now includes the "minimum requirements of life in society" and "living together". This tends to show that freedom of religion has never been considered as an ordinary freedom among the rights guaranteed by our Constitution. 
L e propre des juristes à qui l'on reproche si souvent d'être conservateurs est au contraire de savoir adapter avec une habileté consommée leur vocabulaire figé aux nécessités du temps, pour le meilleur ou pour le pire. Le sens des mots doit toujours être compris au moment où on les emploie. Il arrive ainsi que des concepts immémoriaux rarement utilisés envahissent soudainement la sphère du droit, parce qu'ils correspondent à un besoin actuel. C'est le cas de la dignité qui depuis le XIX ${ }^{\mathrm{e}}$ siècle semblait devoir réserver ses apparitions pour les grandes occasions, par exemple l'abolition de l'esclavage en 1848, qualifié par le décret de Victor Schœlcher d' «attentat contre la dignité humaine », ou encore la proclamation, un siècle plus tard, de la Déclaration universelle des droits de l'homme qui réaffirme la dignité de tous les êtres humains au lendemain de la grande hécatombe. Aujourd'hui, la dignité a tellement envahi le langage de la politique et du droit qu'elle s'est diluée au point qu'on ne sait plus très bien ce qu'elle signifie exactement.

C'est le cas également de l'ordre public, auquel est consacré le présent dossier. On ne compte plus les colloques, les articles ou encore les thèses consacrés depuis une bonne vingtaine d'années à ce concept d'ordre public, qui se diffuse dans toutes les branches du droit et dont on se perd en conjectures sur le sens qu'il convient de lui donner. En tout état de cause, s'intéresser comme on a voulu le faire ici à l'ordre public dans la sphère religieuse, c'est opérer une forme de retour aux sources puisque c'est à la matière religieuse, si importante pour les révolutionnaires, que l'ordre public doit son entrée dans le droit moderne, à l'article 10 de la Déclaration des droits de l'homme et du citoyen (DDHC): «Nul ne doit être inquiété pour ses opinions, même religieuses, pourvu que leur manifestation ne trouble pas l'ordre public établi par la loi.»

\section{L'ARTICLE 10 DE LA DÉCLARATION DES DROITS DE L'HOMME ET DU CITOYEN}

Pour les hommes de cette époque, la notion de l'ordre public doit être comprise au sens littéral, c'est-à-dire comme Hauriou le dira par la suite «l'ordre maintenu dans le public, la paix publique assurée, [qui] s'oppose au désordre et aux perturbations ${ }^{1} »$. Ainsi que l'énonce le Code des délits et des peines du 3 brumaire an IV, l'ordre public est l'objet même du pouvoir de police: «La police est instituée pour maintenir l'ordre public, la liberté, la propriété, la sûreté. » Il s'agit là de ce qu'on appelle communément la police

1. M. Hauriou, Précis de droit administratif et de droit public, Paris, Larose, $4^{e}$ éd. 1901, p. 538. 
administrative, distinguée de la police judiciaire dont la fonction répressive est de rechercher les auteurs d'infractions.

Associée aux «bonnes mœurs», l'expression réapparaît ensuite dans le Code civil de 1804, mais avec une «autre signification» (Hauriou). Entendue dans son sens civiliste, la fonction de l'ordre public est de sanctuariser les lois qui protègent les intérêts généraux de la société, et qui de ce fait s'imposent à tous sans dérogation possible. Ainsi que l'énonce l'article 6 du Code civil, «on ne peut déroger, par des conventions particulières, aux lois qui intéressent l'ordre public et les bonnes mœurs».

Ce qui, à l'article 10 DDHC, retient particulièrement l'attention, c'est que l'ordre public y figure comme la condition de possibilité de la liberté de religion. La manifestation des opinions religieuses, c'est-à-dire concrètement la pratique du culte, ne doit pas «troubler» l'ordre public établi par la loi. Toute la question est alors de savoir s'il s'agit à travers cette disposition d'ériger le catholicisme en religion nationale ou bien si tous les cultes peuvent être pratiqués au grand jour, pour peu que ce soit de manière paisible. Si la question se pose, c'est parce que cette réserve de l'ordre public est réclamée par le haut clergé, en particulier par Monseigneur Gobel, évêque de Lydda, et qu'elle concerne à ses yeux les "non-catholiques ${ }^{2}$ », astreints de la sorte à respecter le culte dominant et relégués pour leurs propres pratiques dans la sphère privée.

Cette vision catholique de l'ordre public est réfutée par Mirabeau, dans une intervention magistrale à l'Assemblée constituante où il expose avec une clarté remarquable sa propre théorie de l'ordre public, la seule qui soit en pleine cohérence avec l'esprit de libéralisme qui inspire la Déclaration ${ }^{3}$. «Le culte», affirme Mirabeau, n'est pas une «chose de police». Il consiste « en prières, en hymnes, en discours, en divers actes rendus à Dieu par des hommes qui s'assemblent en commun; et il est tout à fait absurde de dire que l'inspecteur de police ait le droit de dresser les oremus et les litanies ${ }^{4} »$.

2. Débats de l'Assemblée nationale constituante, séance du 23 août, Archives parlementaires, p. 480. C'est $\mathrm{M}^{\mathrm{gr}}$ Gobel qui est à l'origine de cette référence à l'ordre public dans la Déclaration. Dans son intervention à la Constituante, il explique que les non-catholiques «peuvent être libres dans leurs opinions, même les manifester, sous la seule réserve qu'ils ne troublent pas l'ordre public». Autrement dit, cette réserve de l'ordre public s'impose à ses yeux aux seuls non-catholiques, et elle consiste dans le respect qui est dû au culte dominant.

3. Sur cette question, V. P.-H. PrÉLOt, «L'article 10 de la Déclaration des droits de l'Homme aux origines de la laïcité», in Mélanges en l'honneur de Frédéric Sudre, Paris, Lexis Nexis, 2018, p. 605-615.

4. Archives parlementaires, précit., p. 477. 
Autrement dit, la fonction de l'autorité de police n'est pas de contrôler les croyances ni de protéger un culte particulier au détriment de tous les autres, fût-il le culte «dominant», parce qu'il serait la religion du prince ou celle du plus grand nombre. Sa fonction, ajoute-t-il,

«c'est d'empêcher que personne ne trouble l'ordre et la tranquillité publique. Voilà pourquoi elle veille dans vos rues, dans vos places, autour de vos maisons, autour de vos temples; mais elle ne se mêle point de régler ce que vous y faites; tout son pouvoir consiste à empêcher que ce que vous y faites ne nuise à vos concitoyens.

Je trouve donc absurde encore de prétendre que, pour prévenir le désordre qui pourrait naître de vos actions, il faut défendre vos actions: assurément cela est très expéditif; mais il m'est permis de douter que personne ait ce droit.

Il nous est permis à tous de former des assemblées, des cercles, des clubs, des loges de francs-maçons, des sociétés de toute espèce. Le soin de la police est d'empêcher que ces assemblées ne troublent l'ordre public; mais certes on ne peut imaginer qu'afin que ces assemblées ne troublent pas l'ordre public, il faille les défendre.

Veiller à ce qu'aucun culte, pas même le vôtre, ne trouble l'ordre public, voilà votre devoir; mais vous ne pouvez pas aller plus $\operatorname{loin}^{5}$ ».

Bien entendu, cette notion de l'ordre public n'est absolument pas nouvelle au moment de la révolution, et la police n'est en rien une invention de la modernité libérale. Mais si la réflexion de Mirabeau est aussi fondatrice, c'est parce qu'il s'agit désormais de penser l'ordre public dans une société où, comme l'énonce l'article 2 de la Déclaration, «le but de toute association politique est la sauvegarde des droits naturels et imprescriptibles de l'homme», au premier rang desquels figure la liberté, et notamment la liberté de conscience et d'expression. C'est pour cette raison que l'ordre public des temps nouveaux doit être compris au sens étroit de «l'ordre et la tranquillité publique», à l'exclusion de tout jugement de valeur sur le culte lui-même qui n'est pas comme il le dit «chose de police». Il s'agit là de cet ordre public au contenu minimaliste qu'un siècle plus tard la doctrine qualifiera de «matériel», et qui est celui de l'ordre social libéral.

Mais il faudra du temps encore pour que se réalise la promesse de l'article 10 , puisqu'au XIX ${ }^{e}$ siècle le système napoléonien de la reconnaissance viendra enserrer la pratique publique du culte dans un réseau touffu de prescriptions de police, énoncées notamment dans le Concordat et dans les articles organiques. Quant aux cultes «non reconnus» dans lesquels on

\section{Ibid.}


pourrait être tenté de voir des cultes «libres», ils n'échapperont guère en fait à la surveillance maniaque des autorités publiques ${ }^{6}$.

\section{L'ORDRE PUBLIC DANS LA LOI DE 1905}

L'article $1^{\text {er }}$ de la loi de 1905 reprend dans sa substance l'article 10 DDHC: «La République assure la liberté de conscience. Elle garantit le libre exercice des cultes sous les seules restrictions édictées ci-après dans l'intérêt de l'ordre public.» Ainsi qu'a pu l'observer Charles Benoist, qui siège alors comme député républicain de centre droit, cet article « ressemble à l'article 10 de la Déclaration des droits de 1791, comme le fils ressemble à la mère ${ }^{7} »$. Dans le rapport qu'il présente au nom de la commission, Aristide Briand souligne, dans un passage souvent cité, le rôle phare que cet article $1^{\text {er }}$ est appelé à jouer dans l'avenir:

«Si minutieusement rédigée soit une loi aussi considérable, dont tous les effets doivent être prévus par des dispositions de droit civil, de droit pénal et de droit administratif, elle contient inévitablement des lacunes et soulève des difficultés nombreuses d'interprétation. Le juge saura, grâce à l'article placé en vedette de la réforme, dans quel esprit tous les autres ont été conçus et adoptés. Toutes les fois que l'intérêt de l'ordre public ne pourra être légitimement invoqué, dans le silence des textes ou le doute sur leur exacte application, c'est la solution libérale qui sera la plus conforme à la pensée du législateur. Le libre exercice des cultes tel qu'il est prévu et garanti par le projet réalise un progrès notable dans la voie du libéralisme ${ }^{8}$.»

C'est donc une application libérale de la loi qui est privilégiée, à tout le moins «toutes les fois que l'intérêt de l'ordre public ne pourra être légitimement invoqué ». Comme l'avait fait en son temps la Déclaration, la loi de Séparation pose inévitablement la question de la substance de l'ordre public, qui reviendra régulièrement dans les débats, parce que Briand cède un peu facilement à la tentation d'utiliser l'ordre public comme le mantra qui le dispense d'arguments quand il s'agit de justifier les dispositions contestées du texte qu'il défend. Ainsi qu'a pu le dire Alexandre Ribot, à propos de

6. V. sur cette question la thèse de Franck ZARLEngA, Le régime juridique des Cultes non reconnus dans la France concordataire (1801-1905), thèse, Paris Est, 2018.

7. «Elle et vous, ajoute-t-il à l'intention de Briand, vous limitez également la liberté par l'ordre public »: séance du 11 avril 1905: JO Chambre des députés, p. 1330.

8. A. BRIAND, La séparation des Églises et de l'État. Rapport fait au nom de la commission de la Chambre des députés, Paris, Cornély, 1905, p. 266 [souligné par nous]. 
l'obligation faite aux membres d'une association cultuelle d'être domiciliés dans la commune, au nom de l'ordre public: "Je voudrais bien savoir ce que c'est que l'ordre public. On le fait servir pour justifier un texte, pour se tirer d'embarras ${ }^{9}$.» Mais il ne suffit pas que l'ordre public puisse être invoqué à titre de justification, encore faut-il qu'il le soit «légitimement», ce qui conduit parfois les députés à débattre de ce que les juristes d'aujourd'hui appellent la nécessité et la proportionnalité des mesures envisagées. C'est ainsi que le projet de la commission prohibait initialement les processions et les manifestations religieuses dans l'espace public, afin de prévenir les risques de trouble, alors qu'au contraire ceux qui entendaient sauver les processions soutenaient non sans raison qu'elles se déroulaient la plupart du temps de façon paisible, et qu'il suffisait pour protéger l'ordre public de s'en remettre aux maires du soin d'apprécier la nécessité de les encadrer, voire à titre de mesure extrême de les interdire ponctuellement, mais en considération de circonstances locales qui le justifient et sous le contrôle du juge de la légalité.

Finalement, la gauche républicaine a échoué dans son projet d'interdiction générale des processions, à laquelle les plus anticléricaux de la majorité étaient pourtant viscéralement attachés. Les raisons de cet échec ne sont pas sans intérêt pour le thème de la présente étude, puisque la solution finalement retenue consiste alors à confier aux maires la police des processions et des manifestations religieuses, conformément à la loi municipale de $1884^{10}$. Autrement dit, celles-ci sont soumises au droit commun des manifestations sur la voie publique, c'est-à-dire qu'elles sont autorisées dans le principe, les maires ayant toutefois le pouvoir de les interdire «au nom de l'ordre public», mais sous le contrôle du juge administratif ${ }^{11}$. Ainsi que pourra le dire le

9. 2e séance du 15 juin 1905: JO Chambre des députés, p. 2253.

10. L'article 97 de la loi municipale de 1884 énonce que «la police municipale a pour objet d'assure le bon ordre, la sûreté et la salubrité publique. Elle comprend notamment: $1^{\circ}$ Tout ce qui intéresse la sûreté et la commodité du passage dans les rues, quais, places et voies publiques, ce qui comprend le nettoiement, l'éclairage, l'enlèvement des encombrements, la démolition ou la réparation des édifices menaçant ruine, l'interdiction de rien exposer aux fenêtres ou autres parties des édifices qui puisse nuire par sa chute, ou celle de rien jeter qui puisse endommager les passants ou causer des exhalaisons nuisibles; $2^{\circ}$ Le soin de réprimer les atteintes à la tranquillité publique, telles que les rixes et disputes accompagnées d'ameutement dans les rues, le tumulte excité dans les lieux d'assemblée publique, les attroupements, les bruits et rassemblements nocturnes qui troublent le repos des habitants, et tous actes de nature à compromettre la tranquillité publique; $3^{\circ}$ Le maintien du bon ordre dans les endroits où il se fait de grands rassemblements d'hommes, tels que foires, marchés, réjouissances et cérémonies publiques, spectacles, jeux, cafés, églises et autres lieux publics [...]».

11. «Ne faites pas de distinction entre les manifestations qui ont un caractère religieux et les manifestations qui ont un tout autre caractère. L'ordre public est-il intéressé oui ou 
commissaire du gouvernement Corneille dans ses conclusions à l'arrêt Baldy, en matière de police administrative, «la liberté est la règle, et la restriction de police l'exception ${ }^{12} »$. La solution finalement retenue consacre ainsi le principe selon lequel, dans le régime de séparation, l'activité religieuse n'est pas traitée différemment des autres activités sociales. À cela, on pourrait objecter que la loi de 1905 comprend un titre V spécifique intitulé «Police des cultes » (art. 25 à 36), mais à y regarder de près les dispositions en cause sont pour l'essentiel des mesures d'organisation ${ }^{13}$ ainsi que des dispositions pénales ponctuelles ${ }^{14}$ qui ne constituent pas à proprement parler un régime de police au sens où on l'entend en droit administratif ${ }^{15}$. La police des cultes, dans le nouveau système de la séparation, c'est pour l'essentiel la police de l'ordre public appliquée aux cultes.

\section{LE CARACTÈRE STRICTEMENT «MATÉRIEL » DE L'ORDRE PUBLIC}

Pour la doctrine du droit administratif qui se construit sous la Troisième République, cet article 97 de la loi de 1884 est appelé à jouer un rôle central, car c'est lui désormais qui définit la substance de l'ordre public. À la question de savoir «qu'est-ce que l'ordre public », Louis Rolland peut ainsi répondre ${ }^{16}$ $q^{\prime}$ '« en elle-même, cette notion est extrêmement vague», mais que «des textes permettent de le préciser. Ce sont ceux relatifs à la police municipale. Il résulte de l'article 97 de la loi du 5 avril 1884 [...] que la police a pour objet le bon ordre, c'est-à-dire la tranquillité publique, la sécurité publique, la salubrité publique. Assurer l'ordre public, c'est en somme, assurer ces trois choses. L'ordre public, c'est tout cela, ce n'est que cela».

non? Voilà toute la question. Restons dans la question libérale, dans la solution vraie; c'est celle qu'indique l'amendement, c'est celle de la loi municipale de 1884 qui a été votée par une Chambre républicaine. Nous pouvons bien maintenir le droit aux maires, et aussi aux préfets, au nom de l'ordre public - mais seulement au nom de l'ordre public d'interdire les manifestations qui pourraient devenir dangereuses.»: Alexandre Ribot, $2^{\mathrm{e}}$ séance du 26 juin 1905: JO Chambre des députés, p. 2490.

12. CE, 10 août 1917, Baldy.

13. Régime des réunions pour l'exercice d'un culte, régime des sonneries de cloches, signes ou emblèmes religieux.

14. Art. 29 à 35.

15. À quoi il faut ajouter que ce titre inclut le fameux article 27 qui renvoie au droit commun de la loi de 1884 pour «les cérémonies, processions et autres manifestations extérieures d'un culte».

16. Précis de droit administratif, Paris, Dalloz, 9e éd. 1947, p. 399. 
Si pour Louis Rolland l'ordre public «ce n'est que cela», c'est parce que comme Hauriou il estime que la police ne doit pas s'occuper de morale, sauf à prendre le risque d'attenter à la liberté de conscience et d'opinion. «La police, affirme ce dernier, n'essaie point d'atteindre les causes profondes du mal social, elle se contente de rétablir l'ordre matériel [...]; en d'autres termes, elle ne poursuit pas l'ordre moral dans les idées et dans les sentiments, elle ne pourchasse pas les désordres sociaux, elle est pour cela radicalement incompétente ${ }^{17}$. » La morale publique, et plus globalement ce qu'on appelle aujourd'hui la défense des valeurs ${ }^{18}$, n'est pas en soi un but de police. Ce n'est que dans l'hypothèse où l'atteinte à la morale ou à la décence produirait localement un trouble à l'ordre public que l'activité en cause pourra être ponctuellement interdite ${ }^{19}$. Fondée sur quelques cas d'espèce accumulés au fil du temps, la doctrine a ainsi forgé une sorte de théorie de l'accessoire dont l'effet était de réintégrer à la marge la protection de la moralité publique dans les fonctions de la police.

Cette conception libérale d'un ordre public uniquement préoccupé des troubles extérieurs sera fragilisée par l'arrêt d'assemblée de 1995 dit du «lancer de nain ${ }^{20}$ », dans lequel le Conseil d'État fait du respect de la dignité de la personne humaine «une des composantes de l'ordre public» justifiant l'interdiction d'un spectacle public, y compris « en l'absence de circonstances locales particulières ». Si la décision, pour laquelle aucune question touchant à la liberté de religion n'était en jeu, est à ce jour demeurée relativement isolée, il reste qu'elle est venue fragiliser la vieille conception d'un ordre public purement matériel reprise à l'unisson depuis un siècle par la grande majorité des juristes. En prescrivant, au nom de l'ordre public, une interdiction générale de prêter son corps pour un spectacle jugé attentatoire à la dignité humaine, elle préfigure la loi de 2010 interdisant la dissimulation du visage dans l'espace public ${ }^{21}$.

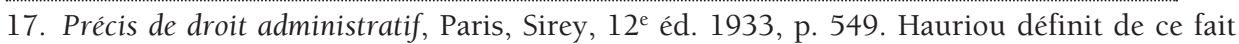
l'ordre public comme un simple «ordre matériel et extérieur considéré comme un état de fait opposé au désordre».

18. Celle-ci relève non pas de l'État, incompétent en tant que puissance publique, mais de ce qu'Hauriou appelle au sens large les «institutions » qui structurent la société à travers la participation des individus et des groupes à l'intérêt collectif.

19. «Si le désordre moral s'extériorise au point de troubler l'ordre public matériel ou d'être pour lui une menace immédiate ou très prochaine, la police administrative est fondée à intervenir »: L. Rolland, Précis de droit administratif, op.cit., p. 400.

20. CE, ass., 27 oct. 1995, Commune de Morsang-sur-Orge.

21. On retiendra que les cas d'invocation de la dignité comme fondement d'une décision administrative d'interdiction sont plutôt rares. Les autres cas emblématiques concernent l'interdiction d'une distribution publique de «soupe gauloise» contenant du porc (CE, 


\section{ORDRE PUBLIC ET LAÏCITÉ}

En normalisant comme elle l'a fait l'ordre public de la sphère religieuse, la loi de 1905 conduisait inévitablement à en banaliser l'usage dans le droit des religions, au point de le reléguer au second plan pendant plus d'un siècle. Avec la séparation, ce n'est plus désormais l'ordre public, dont on ne parle plus guère, mais le concept global de laïcité, ce «néologisme nécessaire» selon la formule de Ferdinand Buisson, qui fixe le cadre dans lequel s'inscrit en France l'exercice de la liberté de religion. Le mot fera son entrée dans le droit au plus haut niveau, avec la Constitution du 27 octobre 1946 énonçant que «La France est une République indivisible, laïque, démocratique et sociale». Une formule reprise à la lettre dans le texte de $1958^{22}$. Les grands débats du $x^{e}$ siècle, que ce soit à propos de l'école privée, du financement des religions sous ses formes les plus diverses, du droit local et en particulier de l'Alsace-Moselle, de l'organisation de l'islam, ou encore de la neutralité des services publics et de ses agents, sont tous placés sous le signe de la laïcité et de ses exigences propres. Dans sa décision du 19 novembre 2004, Traité établissant une Constitution pour l'Europe, le Conseil constitutionnel pourra ainsi souligner la nécessité qu'il y a en France de «concilier» la liberté de culte que garantit à son niveau le traité «avec le principe de laïcité ${ }^{23} »$. La hiérarchie est clairement établie entre une laiicité constitutionnelle immuable et une liberté de culte qui en tout état de cause devra se «concilier» avec elle.

Ayant souligné l'importance du principe de laïcité pour l'aménagement concret du principe de liberté de religion, il revenait au Conseil constitutionnel à en définir les contours, et c'est ce qu'il a fait dans sa décision QPC du 21 février 201324. Dans cette décision, il précise ainsi à propos de la laïcité

ord. réf., 5 janv. 2007, Solidarité des Français), ainsi que celle des spectacles de Dieudonné (V. notamment CE, ord. réf., 9 janv. 2014). Pour le reste, c'est surtout dans le contentieux de la détention qu'il est fait usage de la notion de conditions attentatoires à la dignité humaine.

22. À l'article 2, devenu en 1995 l'article $1^{\text {er }}$.

23. Cons. const., 19 nov. 2004, no 2004-505 DC, Traité établissant une Constitution pour l'Europe. Dans cette décision, le Conseil constitutionnel mentionne également «les dispositions de l'article $1^{\text {er }}$ de la Constitution aux termes desquelles "la France est une République laïque", qui interdisent à quiconque de se prévaloir de ses croyances religieuses pour s'affranchir des règles communes régissant les relations entre collectivités publiques et particuliers $»$.

24. Cons. const., 21 févr. 2013, n 2012-297 QPC, Association pour la promotion et l'expansion de la laïcité [Traitement des pasteurs des églises consistoriales dans les départements du Bas-Rhin, du Haut-Rhin et de la Moselle]. 
constitutionnelle «qu'il en résulte la neutralité de l'État; qu'il en résulte également que la République ne reconnaît aucun culte; que le principe de laïcité impose notamment le respect de toutes les croyances, l'égalité de tous les citoyens devant la loi sans distinction de religion et que la République garantisse le libre exercice des cultes; qu'il implique que celle-ci ne salarie aucun culte ». On retrouve dans cette formulation les principes énoncés aux articles 1 et 2 de la loi de 1905 (qui ne sont pas mentionnés), à l'exception de l'interdiction faite à la République de subventionner les cultes, qui pour le Conseil constitutionnel a valeur simplement législative. Quant au fait que le libre exercice des cultes s'inscrit nécessairement, comme l'indique l'article $1^{\mathrm{er}}$, dans les limites de l'ordre public, il est également consacré au plus haut niveau, si l'on considère que l'ordre public constitue pour le Conseil constitutionnel un « objectif à valeur constitutionnelle ${ }^{25}$ » justifiant les restrictions nécessaires à l'exercice paisible des libertés.

C'est comme l'on sait la controverse relative aux signes religieux et notamment au foulard porté par les musulmanes, à la fin des années 1980, qui va marquer le retour en force de l'ordre public dans le débat relatif à la présence de plus en plus contestée des religions dans l'espace commun. La question de l'extériorisation des croyances ayant été résolue dans un sens libéral par la loi de 1905, qu'il s'agisse des manifestations extérieures collectives ${ }^{26}$ ou du port du costume religieux ${ }^{27}$, la laïcité, définie comme un principe d'organisation des pouvoirs publics, ne pouvait être invoquée comme une justification pertinente pour interdire par voie de mesure générale le port de signes religieux par les individus. Si, avec la loi du 15 mars 2004, c'est malgré tout le principe de laïcité qui a finalement prévalu ${ }^{28}$, c'est parce que l'interdiction concernait alors les seuls établissements du service public scolaire $^{29}$, la discussion ayant porté sur le point de savoir si l'exigence de laïcité, qui s'impose aux personnels, aux locaux ainsi qu'aux programmes, peut également être étendue aux élèves-usagers. Cependant la lecture du rapport de la commission Stasi, à l'origine de la loi, nous révèle que les

25. Cons. const., 20 janv. 1981, $\mathrm{n}^{\circ}$ 80-127 DC, Loi renforçant la sécurité et protégeant la liberté des personnes.

26. On renvoie ici aux discussions évoquées supra à propos des cérémonies, processions et autres manifestations extérieures d'un culte (art. 27).

27. V. la discussion du fameux «amendement Chabert» lors des débats du 26 juin 1905.

28. La loi du 15 mars 2004 est désignée comme la loi «encadrant, en application du principe de laïcité, le port de signes ou de tenues manifestant une appartenance religieuse dans les écoles, collèges et lycées publics» [souligné par nous].

29. À l'exclusion des établissements privés sous contrat. 
nécessités de l'ordre public ont pu également être avancées par la commission pour justifier le vote d'une loi d'interdiction ${ }^{30}$.

Mais si le principe de laïcité pouvait être convoqué pour autoriser une interdiction des signes religieux «ostensibles» dans le cadre scolaire, en revanche il ne pouvait en aucun cas justifier une interdiction vestimentaire applicable dans l'ensemble de l'espace public. C'est pourquoi la réflexion sur les fondements possibles d'une interdiction du port du niqab et de la burqa, dont l'effet est de masquer le visage des femmes qui le portent aux yeux des tiers, a très vite écarté le principe de laïcité, ainsi que les principes d'égalité des sexes et de dignité $e^{31}$, pour retenir comme unique fondement pertinent les exigences de l'ordre public.

\section{L'ÉMERGENCE D'UN NOUVEL ORDRE PUBLIC}

Ce retour en force de l'ordre public, que préfigurait le fameux arrêt du lancer de nain, s'est opéré au prix d'une extension considérable de ses territoires. Selon la définition classique de l'ordre public issue de la tradition républicaine, celui-ci se caractérisait ainsi qu'on a pu le dire comme un ordre purement "matériel», qui ne cherche pas à défendre des valeurs ou à prescrire des comportements sociaux déterminés, en particulier dans des domaines relevant de l'intime tels que la manière de se vêtir ou d'affirmer

30. «La commission, après avoir entendu les positions des uns et des autres, estime qu'aujourd'hui la question n'est plus la liberté de conscience, mais l'ordre public. Le contexte a changé en quelques années. Les tensions et les affrontements dans les établissements autour de questions religieuses sont devenus trop fréquents. Le déroulement normal des enseignements ne peut plus être assuré. Des pressions s'exercent sur des jeunes filles mineures, pour les contraindre à porter un signe religieux. L'environnement familial et social leur impose parfois des choix qui ne sont pas les leurs. La République ne peut rester sourde au cri de détresse de ces jeunes filles. L'espace scolaire doit rester pour elles un lieu de liberté et d'émancipation [...]»: COMMISSION DE RÉFLEXION SUR L'APPLICATION DU PRINCIPE DE LAḮITÉ DANS LA RÉPUBliQue, Rapport au président de la République, 11 déc. 2003, p. 58.

31. Dans son intervention du 22 juin 2009 devant le Congrès, en application de la toute nouvelle procédure de l'article 18 de la Constitution, Nicolas Sarkozy avait déclaré que «le problème de la burqa n'est pas un problème religieux, c'est un problème de liberté, de dignité de la femme. Ce n'est pas un signe religieux, c'est un signe d'asservissement, c'est un signe d'abaissement», ajoutant qu' « elle ne sera pas la bienvenue sur le territoire de la République». Le discours de Nicolas Sarkozy avait été au point de départ du processus législatif d'interdiction. Mais très vite les fondements invoqués par le président de la République (liberté et dignité de la femme) ont été écartés, notamment parce que dans beaucoup de cas le port de la burqa est un choix des femmes elles-mêmes, et non une contrainte imposée par les hommes. 
ses convictions religieuses personnelles. Mais si le port du niqab ou de la burqa dans la rue peut être de nature à générer ponctuellement des risques pour l'ordre public, s'agissant par exemple de l'accès aux banques ou aux espaces sécurisés, en revanche le fait de le porter paisiblement ne génère en soi, la plupart du temps, aucune atteinte objective à l'ordre public, si on veut bien laisser de côté ici le sentiment de gêne ou d'indisposition que pourront ressentir les passants. C'est cette impossibilité de fonder une interdiction générale du niqab ou de la burqa sur l'ordre public entendu en son sens classique qui est à l'origine de l'émergence d'une conception «juridiquement inédite $^{32} »$ de l'ordre public, explicitée par le Conseil d'État dans son Étude relative aux possibilités juridiques d'interdiction du port du voile intégral, rédigée à la demande d'un Premier ministre soucieux de donner un fondement pertinent à son projet d'interdiction.

Ainsi que l'explique le Conseil d'État, l'ordre public ainsi défini

« répond à un socle minimal d'exigences réciproques et de garanties essentielles de la vie en société, qui [...] sont à ce point fondamentales qu'elles conditionnent l'exercice des autres libertés, et qu'elles imposent d'écarter, si nécessaire, les effets de certains actes guidés par la volonté individuelle. Or, ces exigences fondamentales du contrat social, implicites et permanentes, pourraient impliquer, dans notre République, que, dès lors que l'individu est dans un lieu public au sens large, c'est-à-dire dans lequel il est susceptible de croiser autrui de manière fortuite, il ne peut ni renier son appartenance à la société, ni se la voir déniée, en dissimulant son visage au regard d'autrui au point d'empêcher toute reconnaissance [...] Une telle conception, formalisée, aboutirait ainsi, de manière inédite, à retenir une définition "positive" de l'ordre public non plus seulement comme "rempart" contre les abus procédant de l'exercice sans limites des libertés, mais comme le socle d'exigences fondamentales garantissant leur libre exercice ${ }^{33}$.

Bien qu'elle ait été envisagée avec réticence par le Conseil d'État, c'est cette «définition positive ${ }^{34}$ » de l'ordre public qui va servir de fondement juridique à la loi du 11 octobre 2010 interdisant la dissimulation du visage dans l'espace public. Si en effet le législateur a cru devoir prohiber la dissimulation du visage c'est, comme l'explique le Conseil constitutionnel, parce qu'il «a estimé que de telles pratiques peuvent constituer un danger

32. France. Conseil d'État, Étude relative aux possibilités juridiques d'interdiction du port du voile intégral, 2010, p. 26.

33. Ibid., p. 27.

34. Ibid. 
pour la sécurité publique et méconnaissent les exigences minimales de la vie en société».

Cette loi du 11 octobre 2010 a suscité des réactions pour le moins mitigées chez les juristes, parce qu'elle remettait directement en cause un des principes les plus essentiels de l'ordre social démocratique, qui est la liberté de l'espace commun. Des notions au contenu indéterminé telles que les «exigences minimales de la vie en société » ou encore le «vivre ensemble», apparaissent en effet bien trop vagues, bien trop imprécises, dans une société fondée sur le pluralisme et le respect inconditionnel de l'altérité, pour que leur nocivité potentielle ne saute pas aux yeux le jour où un pouvoir moins soucieux des libertés individuelles prétendra s'occuper sérieusement d'ordonner les comportements sociaux. Mais outre qu'elle a été validée sans excès de motivation par le Conseil constitutionnel ${ }^{35}$, et de façon plutôt consensuelle par la Cour de Strasbourg ${ }^{36}$, la loi a été accueillie favorablement par l'opinion publique, et elle est aujourd'hui plébiscitée par une majorité de citoyens qui voient en elle un rempart contre l'islamisme et partant une affirmation de la laïcité ${ }^{37}$. De fait, même si la loi prohibe indistinctement la dissimulation du visage sans jamais nommer le voile intégral, son objet est indiscutablement religieux, et c'est au reste ce qui explique le consensus dont elle est l'objet, alors même que le principe d'un ordre public prescriptif des comportements sociaux, tel que le décrit l'article 6 du Code civil qui l'associe aux «bonnes mœurs», tend à reculer au nom de l'autonomie individuelle.

À ce point d'une histoire qui n'a pas fini de s'écrire, ce rapide survol de deux siècles montre à quel point l'ordre public peut être sollicité dès lors qu'est en cause l'expression des religions dans l'espace social. Tout l'effort de la tradition libérale, en vue d'inscrire l'ordre public des activités religieuses dans le droit commun de la police administrative, est régulièrement contrarié par une tradition de méfiance sinon d'hostilité envers des activités suspectes de remettre en cause l'unité indivisible de la communauté nationale, qui est

35. Outre que le texte a fait l'objet d'une saisie «blanche» destinée à prévenir d'éventuelles QPC à venir, le Conseil constitutionnel français n'excelle pas toujours dans l'art de la motivation.

36. L'arrêt S.A.S. de la Cour de Strasbourg a ceci de paradoxal qu'il valide du bout des lèvres la loi française, mais que cette validation est le fait d'une juridiction quasi unanime (à 15 voix sur 17 ).

37. De l'étude d'opinion intitulée «État des lieux de la laïcité en France», réalisée en janvier 2020 pour l'Observatoire de la laïcité, il ressort que pour $50 \%$ des personnes sondées (contre $36 \%$ et $14 \%$ qui ne se prononcent pas), "l'interdiction de dissimuler son visage dans l'espace public a pour fondement la laïcité». 
la matrice du projet politique révolutionnaire. C'est en ce qu'elle a toujours été perçue comme une menace pour l'unité, tout comme au reste la liberté de l'enseignement qui en est le compagnon de route, que la liberté de religion n’a jamais été considérée comme une liberté ordinaire dans le Panthéon des droits que notre Constitution garantit. 